\title{
Influence of forward hadron production on the development of extensive air shower
}

\author{
Nobuyuki Sakurai ${ }^{* \dagger}$ \\ Tokushima University \\ E-mail: nsakurai@icrr.-tokyo.ac.jp
}

\begin{abstract}
Whereas muon in extensive air shower is an observable sensitive to the primary composition and to the hadron interaction properties, the discrepancy between measured and estimated values has not been solved. Using the new data of forward hadron from an accelerator experiment, a hadron interaction model (QGSJET II-04) is modified. Air shower simulated with the modified interaction model develops quickly and produces more muons. But these changes are not enough to solve the discrepancy.
\end{abstract}

35th International Cosmic Ray Conference - ICRC2017

10-20 July, 2017

Bexco, Busan, Korea

* Speaker.

${ }^{\dagger}$ A footnote may follow. 


\section{Introduction}

Recent extensive air shower (EAS) observations have brought the precise energy spectra of ultra high energy cosmic ray (UHECR), and their results clearly have shown sudden drops of the cosmic ray flux around $10^{19.7} \mathrm{eV}[1,2]$. There are thesis as for the mechanism of this rapid dropping, but no established one [3, 4]. One of the big reason of the difficulty of the interpretation of the energy spectrum comes from the uncertainty in the determination of primary UHECR composition. In order to improve this uncertainty, the accurate knowledge of hadron interaction at UHE region is needed.

On the other hand, the serious discrepancies between data and simulation in EAS observations have been reported independently.

Telescope Array (TA) experment based in Utah USA has been observing UHECR since 2007. The detector of TA includes surface particle detector array (SD) [5] and fluorescence detector telescopes (FD) 6 7]. TA group compared the primary cosmic ray energy calculated using FD data and one using SD data, and found that SD energy scale is about $+27 \%$ higher than the FD[1]. Recent TA's studies suggested the distortion of the lateral distribution of secondary particles on the ground. A study of the radial variation of shower front structure above $10^{19.1} \mathrm{eV}$ which was evaluated using "local age parameter" 9] showed a consistency with the iron-induced air shower [8], although TA composition measurement result agreed more closely with the proton than the iron [10]. Another study was the lateral distribution of the SD signal size in the primary energy range $10^{18.8}<E<10^{19.2} \mathrm{eV}[11]$. The discrepancy between measured SD signal size and MC prediction value was increased along with the distance from the shower core. Excessive secondary particle far from shower core in TA data may be an indication of the excess of muons in EAS.

The Pierre Auger Obervatory (PAO) located in Argentina is also the hybrid detector of SD and FD. Their water-Cherenkov type SD could count the number of muons in EAS, and was observed the surplus muon on the ground[12]. While the numbers of electro-magnetic component agreed with the prediction of the hadron interaction models, $33 \%$ more muons were detected than predicted by the EPOS-LHC[13] and 60\% more were detected than predicted by the QGSJET II-04[14]. Their study on the muon production depth showed the quick development of muon component in air shower[15].

It is not known whether these anomalies can be explained by some features of hadron interactions which is not correctly considered, or may be indications of some new phenomenon of physics at UHE region. As proposals of the former case, the sensitivity of the number of muons in EAS to baryon and meson production in hadron collision are discussed in [16] and [17]. Proposals for possible new physics are discussed in [18], [19] and [20].

\section{Information from LHCf experiment}

The important quantities of the hadron interaction which are most relevant for development of EAS are the total inelastic cross section, the secondary particles spectra and inelastisity. The inelastic cross section was measured precisely by TOTEM experiment [21]. The secondary particle spectra was measured by the forward detectors. The inelastisity $k$ is the fraction of energy of 

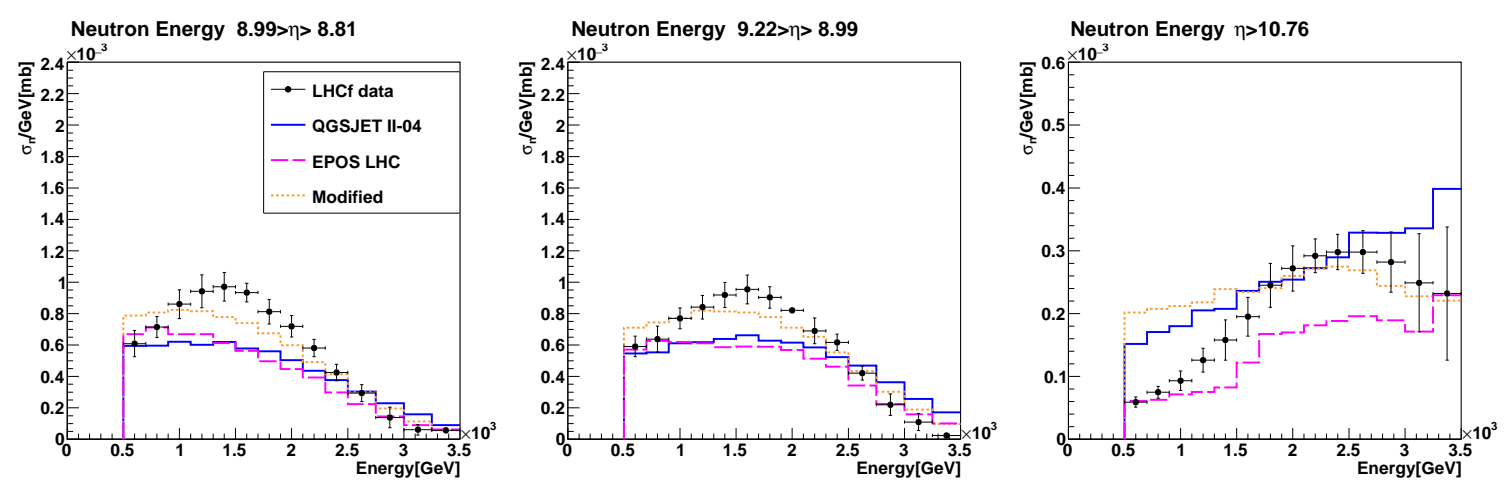

Figure 1: The neutron energy spectra neutron compared to pp collisions at $\sqrt{s}=7 \mathrm{TeV}$ simulated with QGSJET II-04 (blue full line), EPOS-LHC (pink dashed line) and a model modified by this study (orange dotted line).

the primary cosmic rays used to generate secondary particles and is directory measured by the measurement of the forward baryon that provides $1-k$, or elastisity.

The aim of Large Hadron Collider forward (LHCf) experiment [22] is the study of the neutral particle production cross sections in the very forward region of proton-proton and proton-nucleus interactions at the Large Hadron Collider (LHC). The LHCf detectors covered pseudo-rapidity $\eta$ range from 8.7 to $\infty$. So they could measure the peak of energy flux distribution as a function of $\eta$ at $\sqrt{s}=14 \mathrm{TeV}$ proton-proton (pp) collisions which corresponds to the laboratory energy of $10^{17}$ $\mathrm{eV}$ [23].

LHCf group published the inclusive production of neutral pions in pp collisions at $\sqrt{s}=2.76$ and $7 \mathrm{TeV}$ and proton-lead collisions at nucleon-nucleon center-of-mass energy of $\sqrt{s_{N N}}=5.02$ $\mathrm{TeV}$ [24]. In pp collisions, QGSJET II-04 showed an overall better agreement with LHCf data than EPOS-LHC.

LHCf group also published the neutron energy spectra in pp collisions at $\sqrt{s}=7 \mathrm{TeV}$ [25]. DPMJET 3.04 described the LHCf data well at the lower $\eta$ range $(9.22>\eta>8.81)$. Neutron production rate at $\eta>10.76$ predicted by QGSJET II-03 was similar to the LHCf data. However no model perfectly explained the experimental results over the entire pseudo-rapidity range.

\section{Comparison with new hadron interaction models and model modification}

Fig 1 shows the LHCf neutron spectra together with the model predictions which include the contributions of other hadrons such as $\Lambda \mathrm{s}$ and $K_{s}^{0} \mathrm{~s}$. In the most forward pseudo-rapidity range, QGSJET II-04 model predicts higher neutron production rate than the experimental result. However, the prediction of QGSJET II-04 is clearly smaller than the measured neutron production rate in the smaller pseudo-rapidity range.

The total energy carried by forward neutrons as a function of pseudo-rapidity is shown in the left panel of Fig 2 Curves show the predictions of hadron interaction models which take the energy threshold of LHCf neutron result $(E>500 \mathrm{GeV})$. Each cross symbol is the energy flux calculated from published LHCf data. Predictions of QGSJET II-04 is relatively similar to data, but is still small around the peak of energy flow distribution. The right panel of Fig 2 shows the $\pi^{0}$ energy 

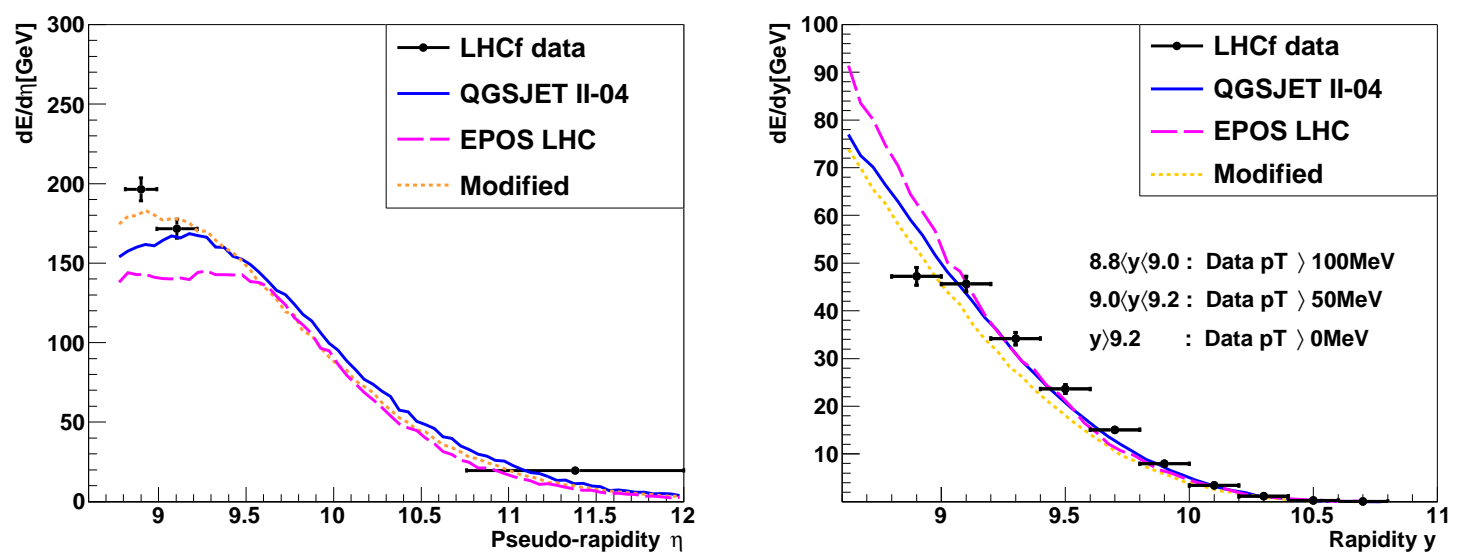

Figure 2: The energy flux of neutron (left) and $\pi^{0}$ (right) as measured by LHCf collaboration, compared to pp collisions at $\sqrt{s}=7 \mathrm{TeV}$ simulated with QGSJET II-04 (blue full line), EPOS-LHC (pink dashed line) and a model modified by this study (orange dotted line). The simulation curves of $\pi^{0}$ does not take the effect of $p T$ threshold into account.

flux as a function of rapidity in $\sqrt{s}=7 \mathrm{TeV}$ pp collisions. For the $\pi^{0}$, all models are consistent with data above $y>9.2$.

To increase baryon energy flow at very forward region, I make a trial of increasing the events whose leading particle is $\Lambda$. The developed method is as follows.

1. Select events whose leading particles are not nucleon.

2. A part of these events are converted to events whose leading particles are lambda. The probability of event conversion $\left(P_{\text {conv }}\right)$ is set at 0.445 .

Neutron energy spectra and energy flux which are modified by this method is also shown in the left panel of Fig.2. Shifting of peak position to lower pseudo-rapidity is related to the increasing the inelasticity of each collision. The $\pi^{0}$ energy flux of the modified model is shown in the right panel of Fig 2. At the very forward region, difference between original QGSJET II-04 and the modified model is very large.

\section{Air Shower Simulation}

To study the importance of the forward neutron energy flux for the interpretation of EAS observations, I modified hadron interaction during EAS simulation as described above. In the following, all EAS simulations are performed for primary cosmic ray energy of $10^{19 \sim 10^{20}} \mathrm{eV}$ with Conex v4r37 [26, 27]. QGSJET II-04 was used as a high energy hadron interaction model in the MC part.

The impact of the modification of forward baryon energy spectrum is as shown in Fig 3 Modified model predicts the smaller shower maximum position than original one. With this modified model, forward neutron spectrum becomes more softer than the predictions of current hadron interaction models. Then inelastisity becomes larger so that air shower develops more quickly than the 

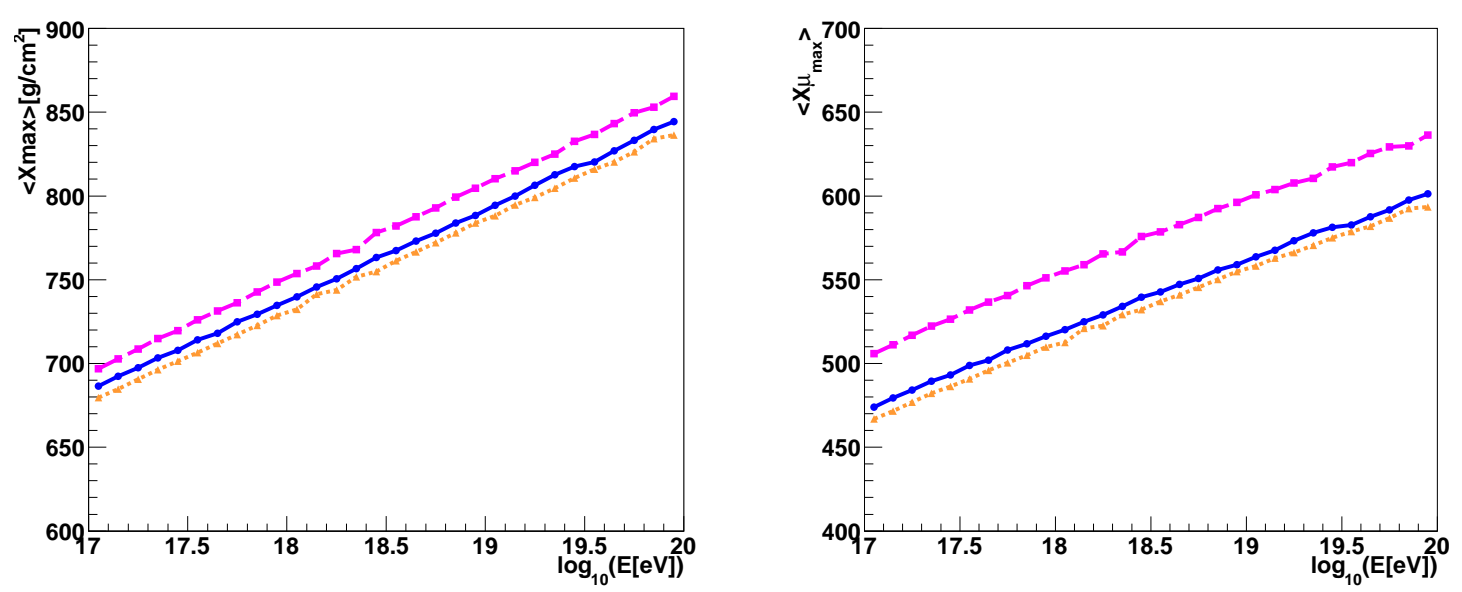

Figure 3: Primary energy dependence $X_{\max }$ and $X_{\max }^{\mu}$ of proton induced showers simulated with QGSJET II-04 and EPOS-LHC at primary energy $E=10^{17} \sim 10^{20} \mathrm{eV}$. The proton induced shower with the hadron interaction model modified by this study (orange dotted line) is also shown.

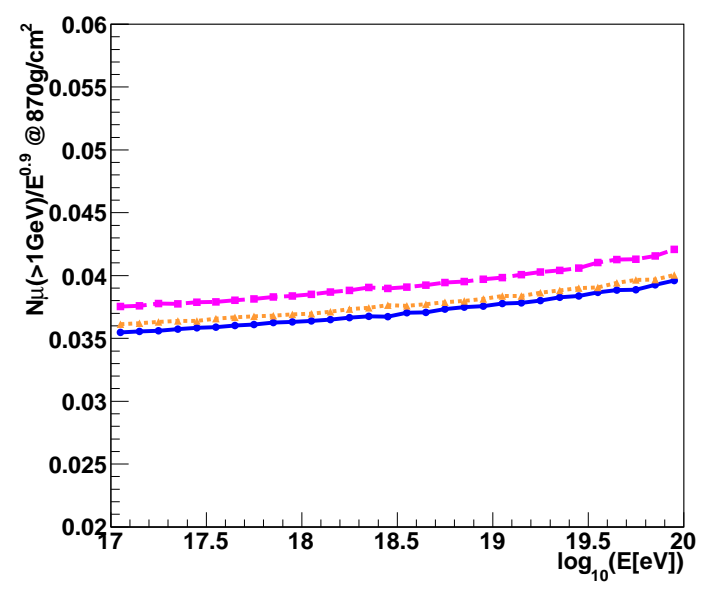

Figure 4: The averaged secondary particle distribution at TA detector level $\left(875 \mathrm{~g} / \mathrm{cm}^{2}\right)$. Proton (red), carbon(Green) and iron (blue) induced showers simulated with QGSJET II-04 at primary energy $E=10^{17} \sim$ $10^{20} \mathrm{eV}$. The proton induced shower with the hadron interaction model modified by this study (orange) is also shown.nction of primary energy and the right is the number density of muons above $1 \mathrm{GeV}$.

simulation with original interaction model. The total number of muons on the ground is increased. The baryons generated to the forward region is strongly related to the number of muons observed on the ground. Unlike $\pi_{0}$ s which decay immediately into two photons and give the energy to the electromagnetic cascade, baryons can still interact and produce charged pions which then decay into muons. 


\section{Conclusion}

QGSJET II-04 is modified so as the excess of forward neutron energy flow in LHCf result is reproduced. Using the modified model, the maximum depth of proton induced shower is smaller than the prediction of the original model. The total number of muon on the ground is increased, but the number density far from shower core does not changed.

\section{Acknowledgments}

This work was supported by JSPS KAKENHI Grant Number JP15K05102.

\section{References}

[1] T. Abu-Zayyad et al., Astrophys. J. Lett., 768:L1 (2013)

[2] J. Abraham et al., Phys. Lett B, 685, 239 (2010)

[3] E. Kido and O.E. Kalashev, for Telescope Array Collaboration in Proc. 34th ICRC 2015258

[4] A. di Matteo, for Pierre Auger Collaboration in Proc. 34th ICRC 2015249

[5] T. Abu-Zayyad et al., Nucl. Instrum. Meth. A 689, 87 (2013)

[6] T. Abu-Zayyad et al., Nucl. Instrum. Meth. A 676, 54 (2012)

[7] T. Abu-Zayyad et al., Astropart. Phys. 109, 39 (2012)

[8] N. Sakurai, for Telescope Array Collaboration in Proc. 33th ICRC 2013130

[9] J. N. Capdevielle and J. Gawin J. Phys. G (Nucl. Phys.) 8, 1317 (1982)

[10] R.U. Abbasi et al., Astropart. Phys. 64, 49 (2015)

[11] R. Takeishi, Doctral thesis, The University of Tokyo (2016)

[12] A. Aab et al., Phys. Rev. Lett., 117, 192001 (2016)

[13] T. Pierog, Iu Karpenko, J.M. Katzy, E. Yatsenko and K. Werner, Phys. Rev. C 92, 034906 (2015)

[14] S. Ostapchenko, Phys. Rev. D 83, 014018 (2011)

[15] A. Aab et al., Phys. Rev. D 90, $012012+039904$ (2014)

[16] T. Pierog, Proc. ISVHECRI 2012, EPJ Web Conf. 5203001 (2013)

[17] H.J. Drescher, Phys. Rev. D 77056003 (2008)

[18] J. Allen and G. Farrar, arXiv:1307.7131

[19] G.R. Farrar and J. Allen, arXiv:1307.2322

[20] J. Alvarez-Muniz et al., arXiv:1209.6474

[21] G. Antchev et al., Europhys. Lett. 10121003 (2013)

[22] O. Adriani et al., JINST 3 S08006 (2008)

[23] T. Sako, Proc. KMI Inaguration Conference on Quest for the Origin of particles and the Universe(KMIIN), 112-118 (2011) 
[24] O. Adriani et al., Phys. Rev. D 94, 032007 (2016)

[25] O. Adriani et al., Phys. Lett. B, 750, 360 (2015)

[26] T. Bergmann et al., Astropart. Phys 26, 420 (2007)

[27] T. Pierog et al., Nucl. Phys. Proc. Suppl. 151159 (2006)

[28] A. Ferrari, P.R. Sala, A. Fasso and J. Ranft, CERN-2005-10 (2005), INFN/TC_05/11, SLAC-R-773

[29] T.T. Bohlen, F. Cerutti, M.P.W. Chin, A. Fasso, A. Ferrari, P.G. Ortega, A. Mairani, P.R. Sala, G. Smirnov, and V. Vlachoudis, Nuclear Data Sheets, 120, 211-214 (2014) 